\title{
Electro-Mechanical Coupling Properties of Band Gaps in an Elastic/Piezoelectric Phononic Crystal Nonlocal Nanobeam With Periodically Attached "spring-Mass" Resonators
}

\section{Denghui Qian ( $\nabla$ dhqian@just.edu.cn )}

Jiangsu University of Science and Technology https://orcid.org/0000-0001-5516-6831

Jianchun Wang

China Ship Scientific Research Center

Feiyang He

Nanjing University of Aeronautics and Astronautics

Original Article

Keywords: phononic crystal nanobeam, electro-mechanical coupling effect, nonlocal effect, locally resonant mechanism

Posted Date: January 29th, 2021

DOl: https://doi.org/10.21203/rs.3.rs-155424/v1

License: (c) (1) This work is licensed under a Creative Commons Attribution 4.0 International License.

Read Full License 
Electro-mechanical coupling properties of band gaps in an elastic/piezoelectric phononic crystal nonlocal nanobeam with periodically attached "spring-mass" resonators

\section{Denghui Qian}

School of Naval Architecture \& Ocean Engineering, Jiangsu University of Science and Technology, Zhenjiang 212100, Jiangsu, China

e-mail:dhqian@just.edu.cn

\section{Jianchun Wang}

China Ship Scientific Research Center, Wuxi 214082, Jiangsu, China

\section{Feiyang He}

State Key Laboratory of Mechanics and Control of Mechanical Structures, College of Aerospace Engineering, Nanjing University of Aeronautics and Astronautics, Nanjing 210016, Jiangsu, China

\section{ABSTRACT}

The model of a locally resonant (LR) epoxy/PZT-4 phononic crystal (PC) nanobeam with "spring-mass" resonators periodically attached on epoxy is proposed. The corresponding band structures are calculated by coupling Euler beam theory, nonlocal piezoelectricity theory and plane wave expansion (PWE) method. Three complete band gaps with widest total width less than $10 \mathrm{GHz}$ can be formed in the proposed nanobeam by comprehensively comparing the band structures of three kinds of LR PC nanobeams with resonators attached or not. Furthermore, influencing rules of the coupling fields between electricity and mechanics, "spring-mass" resonator, nonlocal effect and different geometric parameters on first three band gaps are discussed and summarized. All the investigations are expected to be applied to realize the active control of vibration in the region of ultrahigh frequency.

Key words: phononic crystal nanobeam; electro-mechanical coupling effect; nonlocal effect; locally resonant mechanism

\section{INTRODUCTION}

In recent years, kinds of NEMS with excellent properties of small scale, ultrahigh frequency, low energy consumption, high sensitivity and so on, have shown potential applications in wide fields such as national defense, civil use and biomedicine. Presently, the existed piezoelectric nano-devices contain biological motor, resonator sensor, filter, single electron device and so on ${ }^{[1]}$. As is well-known, the most core elements of piezoelectric nano-devices are formed by the basic structures such as piezoelectric nanofilm, piezoelectric nanobeam, piezoelectric nanoplate and so on. Hence, investigations on the mechanical properties such as bending, buckling, vibration and stability of NEMS can be attributed to the corresponding researches on the basic piezoelectric nanostructures. PCs are a sort of man-made composite periodic structures. Recently, different kinds of PCs have gained extensive attention of many researchers because of the characteristics of band gaps, which will be applied to the fields of vibration and noise control, acoustic lens, acoustical device and so on ${ }^{[2]}$. In consequence, based on the common application background in the field of acoustical device, the design ideas between NEMS and PC can be combined to form piezoelectric PC nanostructure, which will show new mechanical properties based on gathering the superior performances of $\mathrm{PC}$ structures, nano materials and piezoelectric materials.

Presently, the direct studies on piezoelectric PC nanostructures were a small amount. Based on transfer matrix (TM) method and nonlocal theory, Chen, Yan and Wang et al. studied the anti-plane transverse wave mode ${ }^{[3]}$, symmetric wave $\operatorname{mode}^{[4]}$ and plane wave mode ${ }^{[5]}$ of one-dimensional (1D) layered piezoelectric PC nanostructures in detail. Miranda Jr and Dos 
Santos ${ }^{[6]}$ calculate the band structures of two-dimensional (2D) piezoelectric PC nanostructures consisting of difform inclusions in kinds of lattices, and research the corresponding properties of band gaps. Based on PWE method, the electro-mechanical coupling bandgap properties of piezoelectric PC nanobeams from the perspectives of surface effects ${ }^{[7]}$, nonlocal effects $^{[8]}$ and nonlinearity ${ }^{[9]}$ were investigated by Qian et al.. Thus, there is still a large space on many research directions related to piezoelectric PC nanostructures

Recently, the mechanical properties of different piezoelectric nanostructures and bandgap properties of various PC structures related to piezoelectric PC nanostructures have been studied widely. Because of size dependence, theory and experiment have proved that classical continuum elasticity theory maturely and widely applied to macrostructures cannot be used to structures at nanoscale. However, some higher-order theories based on the revision of classical continuum elasticity theory have been developed, such as: nonlocal elasticity ${ }^{[3-5,8,9]}$, surface elasticity ${ }^{[7]}$, couple stress elasticity ${ }^{[10]}$ and strain gradient elasticity theories ${ }^{[1]]}$. Particularly, nonlocal elasticity theory was further developed into nonlocal piezoelectricity theory, which have been widely used for researching the different mechanical characteristics of kinds of piezoelectric nanostructures ${ }^{[12,13]}$. PC structures macroscopically have been proposed and researched widely for more than thirty years. Particularly, in order to meet engineering requirements, the mentalities of designing of various PCs have been introduced to several basic elastic elements, for instance: rod, beam, plate and so on ${ }^{[14,15]}$. However, the main disadvantage of such PC structures is the uncontrol of band gaps if they are manufactured, which can be overcome by the appearance of some new type PC structures based on the coupling of multi-physics, for example: magneto-electro-elastic PCs, piezoelectric PCs, piezomagnetic PCs and so on ${ }^{[16-18]}$. Among them, band gaps of piezoelectric PCs can be effectively adjusted and controlled by means of converting electric and mechanical fields. The orders of magnitude of frequency range of band gaps in the above-mentioned macroscopic PCs are generally from hertz $(\mathrm{Hz})$ to megahertz $(\mathrm{MHz})$. But recently, with the quick growth of nanotechnology in different research areas, studies on PC nanostructures have also been appeared in large numbers ${ }^{[19,20]}$, and the orders of magnitude of frequency range of band gaps are hoiked into gigahertz $(\mathrm{GHz})$ even terahertz $(\mathrm{THz})$, which makes the control of vibration in ultrahigh frequency region possible.

According to the existing researches about piezoelectric PCs, PC nanostructures and piezoelectric nanostructures, the band structures of proposed LR epoxy/PZT-4 PC nanobeams are calculated and obtained by combining and implementing Euler beam and nonlocal piezoelectricity theories to PWE method in this paper. Moreover, for the sake of revealing the bandgap characteristics, influencing rules of the coupling fields between electricity and mechanics, "spring-mass" resonator, nonlocal effects and geometric sizes on band gaps of first three orders are investigated.

\section{MODEL AND METHOD}

\section{A. LR elastic/piezoelectric PC Euler nonlocal nanobeam}

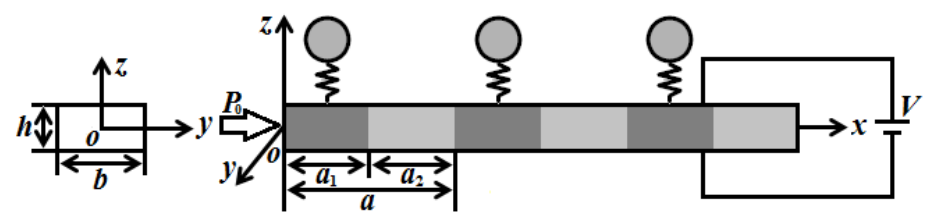

FIG. 1. Model of LR elastic/piezoelectric PC nanobeam.

As shown in Fig. 1, dark gray and light gray are used to express piezoelectric material PZT-4 
and elastic material epoxy, respectively. By means of repeating such two different materials periodically along $x$-direction, and attaching the midpoint of each PZT-4 with a "spring-mass" resonator along $z$-direction, the model of a LR elastic/piezoelectric PC nanobeam is formed. As displayed, the entire nanobeam is applied by an axial force $P_{0}$ and each PZT-4 is applied by an electrical voltage $V$, which the electrical voltage of only one PZT-4 is presented in the figure. The length of each PZT-4 and that of each epoxy in an unit cell are denoted by $a_{1}$ and $a_{2}$ respectively, which can be applied to express the lattice constant of PC nanobeam as $a=a_{1}+a_{2}$ and the position of each resonator as $X=a_{1} / 2+\bar{n} a(\bar{n}=0, \pm 1, \pm 2, \cdots)$. The width and thickness of nanobeam with uniform rectangular cross section are $b$ and $h$ respectively. During the calculations, all of the material parameters can be sought in Table 1.

Table 1. Material parameters of PZT-4 ${ }^{[8]}$ and epoxy.

\begin{tabular}{cccccc}
\hline Material & $\rho / \mathrm{kgm}^{-3}$ & $E / \mathrm{GPa}$ & $c_{11} / \mathrm{GPa}$ & $e_{31} / \mathrm{Cm}^{-2}$ & $\kappa_{33} / \mathrm{CV}^{-1} \mathrm{~m}^{-1}$ \\
\hline PZT-4 & 7500 & & 132 & -4.1 & $7.124 \times 10^{-9}$ \\
epoxy & 1180 & 4.35 & & & \\
\hline
\end{tabular}

The bending vibration equations of the proposed LR elastic/piezoelectric PC Euler nonlocal nanobeam ${ }^{[8,21]}$ are as follows:

where

$$
\left\{\begin{array}{c}
\frac{\partial^{2}}{\partial x^{2}}\left(m(x) \frac{\partial^{2} W(x)}{\partial x^{2}}\right)-n(x) \frac{\partial^{2} W(x)}{\partial x^{2}}+\mu^{2} \frac{\partial^{2}}{\partial x^{2}}\left[n(x) \frac{\partial^{2} W(x)}{\partial x^{2}}\right] \\
-\omega^{2} o(x) W(x)+\omega^{2} \mu^{2} \frac{\partial^{2}}{\partial x^{2}}[o(x) W(x)]=\sum_{X} Q(X) \\
-\omega^{2} m_{\mathrm{R}} W_{\mathrm{R}}(X)=Q_{\mathrm{R}}(X)
\end{array}\right.
$$

$$
\left\{\begin{array}{l}
Q(X)=-k_{\mathrm{R}}\left[W(X)-W_{\mathrm{R}}(X)\right] \delta(x-X) \\
Q_{\mathrm{R}}(X)=k_{\mathrm{R}}\left[W(X)-W_{\mathrm{R}}(X)\right]
\end{array}\right.
$$

Here, $W(x)$ is the bending displacement of entire nanobeam along $z$-direction, $W(X)$ and $W_{\mathrm{R}}(X)$ are the displacements of nanobeam and mass along $z$-direction at position $X$, respectively. $Q(X)$ and $Q_{\mathrm{R}}(X)$ represent the forces applied to nanobeam and mass by spring, separately. Besides, $m_{\mathrm{R}}$ and $k_{\mathrm{R}}$ denote the mass and stiffness of a resonator respectively, $\delta(x-X)$ represents a one-dimensional (1D) delta function, and $\omega$ is the flexural vibration frequency. On the basis of nonlocal piezoelectricity theory, a nonlocal coefficient $\mu$ is introduced to represent the nonlocal effect, and the derived parameter $\eta=\mu / a$ can be further applied to represent the ratio between nonlocal size and lattice constant ${ }^{[8]}$.

Moreover, the derived parameters $\left(m_{1}, m_{2}\right),\left(n_{1}, n_{2}\right)$ and $\left(o_{1}, o_{2}\right)$ (subscripts 1 and 2 denotes materials PZT-4 and epoxy respectively) can be represented uniformly by functions $m(x)$, $n(x)$ and $o(x)$ as shown in in Eq. (1), which are ${ }^{[8]}$ :

$$
\begin{gathered}
m_{1}=\frac{b h^{3}}{12} \bar{c} \\
n_{1}=P_{0}+b e_{31} V \\
o_{1}=\rho_{1} b h \\
m_{2}=\frac{b h^{3}}{12} E \\
n_{2}=P_{0} \\
o_{2}=\rho_{2} b h
\end{gathered}
$$

where $\bar{c}=c_{11}+e_{31}^{2} / \kappa_{33}$, which $c_{11}, e_{31}$ and $\kappa_{33}$ are the constants of elasticity, 
piezoelectricity and dielectricity of PZT-4, separately. $E$ represents the modulus of elasticity of epoxy. In addition, $\rho_{1}$ and $\rho_{2}$ refer to the densities of materials PZT-4 and epoxy, separately.

Based on 1D PWE method and the periodicity of PC nanobeam in $x$-direction ${ }^{[22]}$, functions $m(x), n(x)$ and $o(x)$ are expanded into spatial Fourier series:

$$
\left\{\begin{array}{l}
m(x)=\sum_{G} m(G) e^{i G x} \\
n(x)=\sum_{G} n(G) e^{i G x} \\
o(x)=\sum_{G} o(G) e^{i G x}
\end{array}\right.
$$

where $G$ refers to the $1 \mathrm{D}$ reciprocal-lattice vector.

In Eq. (9), if the surface of PZT-4 is attached by resonators, function $\xi(G)(\xi=m, n, o)$ is expressed as:

$$
\xi(G)=\left\{\begin{array}{lc}
\xi_{1} f+\xi_{2}(1-f), & G=0 \\
\left(\xi_{1}-\xi_{2}\right) \psi(G), & G \neq 0
\end{array}\right.
$$

where $\xi_{1}$ and $\xi_{2}$ respectively represent the relevant parameters of materials PZT-4 and epoxy. $f=a_{1} / a$ denotes the filling ratio of material PZT-4 in a unit cell, $\psi(G)=f \sin \left(G a_{1} / 2\right) /$ $\left(G a_{1} / 2\right)$.

However, if the surface of epoxy is attached by resonators, function $\xi(G)$ is expressed as:

$$
\xi(G)=\left\{\begin{array}{lc}
\xi_{2} f+\xi_{1}(1-f), & G=0 \\
\left(\xi_{2}-\xi_{1}\right) \psi(G), & G \neq 0
\end{array}\right.
$$

where $\xi_{1}$ and $\xi_{2}$ also respectively represent the relevant parameters of materials PZT-4 and epoxy. But $f$ and $\psi(G)$ are changed, which $f=a_{2} / a$ denotes the filling ratio of material epoxy in a unit cell, $\psi(G)=f \sin \left(G a_{2} / 2\right) /\left(G a_{2} / 2\right)$.

On the basis of $1 \mathrm{D}$ periodicity of $\mathrm{PC}$ nanobeam and Bloch theory ${ }^{[21]}, W(x)$ is written as:

$$
W(x)=\sum_{G^{\prime}} W\left(G^{\prime}\right) e^{i\left(k+G^{\prime}\right) x}
$$

where $k$ refers to the 1D Bloch wave vector located at the irreducible first Brillouin zone (1BZ).

Besides, the periodicity and Bloch theory also suggest the following relations:

$$
\left\{\begin{array}{c}
W(X)=W(0) e^{i k X} \\
W_{\mathrm{R}}(X)=W_{\mathrm{R}}(0) e^{i k X}
\end{array}\right.
$$

and the 1D delta function suggests that:

$$
\sum_{X} e^{i k X} \delta(x-X)=e^{i k x} \sum_{X} \delta(x-X)
$$

Define $g(x)=\sum_{X} \delta(x-X)$, which can be expanded to spatial Fourier series:

$$
g(x)=\sum_{G^{\prime}} g\left(G^{\prime}\right) e^{i G^{\prime} x}
$$

where

$$
g\left(G^{\prime}\right)=\frac{1}{a} \int_{a} g(x) e^{-i G^{\prime} x} \mathrm{~d} x=\frac{1}{a} \int_{a} \sum_{X} \delta(x-X) e^{-i G^{\prime} x} \mathrm{~d} x=\frac{1}{a}
$$

Substituting Eq. (16) to (15), it can be obtained that:

$$
\sum_{X} \delta(x-X)=\frac{1}{a} \sum_{G^{\prime}} e^{i G^{\prime} x}
$$

Eq. (12) also suggests that: 


$$
W(0)=\sum_{G^{\prime \prime}} W\left(G^{\prime \prime}\right)
$$

By substituting Eqs. (2), (9), (12), (13), (14), (17) and (18) to (1), it gives that:

$$
\left\{\begin{array}{c}
\sum_{G^{\prime}}\left\{\left(k+G^{\prime \prime}\right)^{2} m\left(G^{\prime \prime}-G^{\prime}\right)\left(k+G^{\prime}\right)^{2}+\left[1+\mu^{2}\left(k+G^{\prime \prime}\right)^{2}\right] n\left(G^{\prime \prime}-G^{\prime}\right)\left(k+G^{\prime}\right)^{2}\right\} W\left(G^{\prime}\right) \\
-\omega^{2} \sum_{G^{\prime}}\left\{\left[1+\mu^{2}\left(k+G^{\prime \prime}\right)^{2}\right] o\left(G^{\prime \prime}-G^{\prime}\right)\right\} W\left(G^{\prime}\right)=-\frac{k_{\mathrm{R}}}{a}\left[\sum_{G^{\prime}} W\left(G^{\prime}\right)-W_{\mathrm{R}}(0)\right] \\
-\omega^{2} m_{\mathrm{R}} W_{\mathrm{R}}(0)=k_{\mathrm{R}}\left[\sum_{G^{\prime}} W\left(G^{\prime}\right)-W_{\mathrm{R}}(0)\right]
\end{array}\right.
$$

If $N$ reciprocal-lattice vectors is picked, Eq. (19) can also be matriculated as:

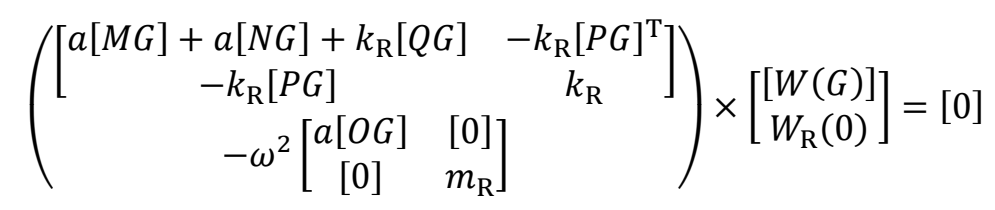

where

$$
\begin{aligned}
& {[M G]_{i j}=\left(k+G_{i}\right)^{2} m\left(G_{i}-G_{j}\right)\left(k+G_{j}\right)^{2}} \\
& {[N G]_{i j}=\left[1+\mu^{2}\left(k+G_{i}\right)^{2}\right] n\left(G_{i}-G_{j}\right)\left(k+G_{j}\right)^{2}} \\
& {[O G]_{i j}=\left[1+\mu^{2}\left(k+G_{i}\right)^{2}\right] o\left(G_{i}-G_{j}\right)} \\
& {[P G]=\left[\begin{array}{llll}
1 & 1 & \cdots & 1
\end{array}\right]_{1 \times N}} \\
& {[Q G]=[P G]^{\mathrm{T}}[P G]}
\end{aligned}
$$

Eq. (20) can be regarded as a representative generalized eigenvalue equation of $\omega^{2}$. By means of iteratively solving such an equation corresponding to each $k$, the band structure of proposed LR elastic/piezoelectric PC Euler nonlocal nanobeam is calculated and obtained.

\section{B. LR piezoelectric PC Euler nonlocal nanobeam}

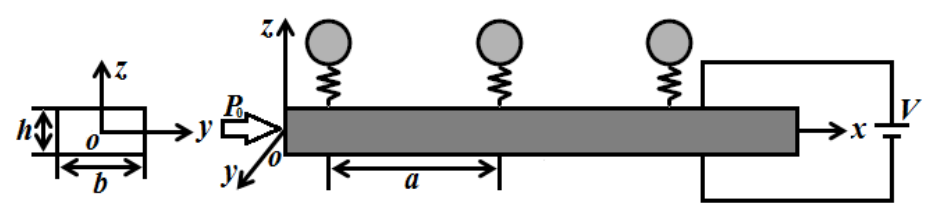

FIG. 2. Model of LR piezoelectric PC nanobeam.

If the elastic material epoxy is ignored and only piezoelectric material PZT-4 is considered in the base beam, a LR piezoelectric PC nanobeam is formed and shown in Fig. 2.

The corresponding bending vibration equations of such a nanobeam can be reduced from Eq. (1) and written as:

$$
\left\{\begin{array}{c}
m_{1} \frac{\partial^{4} W(x)}{\partial x^{4}}-n_{1} \frac{\partial^{2} W(x)}{\partial x^{2}}+\mu^{2} n_{1} \frac{\partial^{4} W(x)}{\partial x^{4}}-\omega^{2} o_{1} W(x)+\omega^{2} \mu^{2} o_{1} \frac{\partial^{2} W(x)}{\partial x^{2}}=\sum_{X} Q(X) \\
-\omega^{2} m_{\mathrm{R}} W_{\mathrm{R}}(X)=Q_{\mathrm{R}}(X)
\end{array}\right.
$$

By substituting Eqs. (2), (12), (13), (14), (17) and (18) to (26), it obtains that: 


$$
\left\{\begin{array}{c}
\left\{m_{1}\left(k+G^{\prime}\right)^{4}+\left[1+\mu^{2}\left(k+G^{\prime}\right)^{2}\right] n_{1}\left(k+G^{\prime}\right)^{2}\right\} W\left(G^{\prime}\right)-\omega^{2}\left[1+\mu^{2}\left(k+G^{\prime}\right)^{2}\right] o_{1} W\left(G^{\prime}\right) \\
=-\frac{k_{\mathrm{R}}}{a}\left[\sum_{G^{\prime}} W\left(G^{\prime}\right)-W_{\mathrm{R}}(0)\right] \\
-\omega^{2} m_{\mathrm{R}} W_{\mathrm{R}}(0)=k_{\mathrm{R}}\left[\sum_{G^{\prime}} W\left(G^{\prime}\right)-W_{\mathrm{R}}(0)\right]
\end{array}\right.
$$

Eq. (27) can also be matriculated to the same form of Eq. (20), the relevant submatrices $[P G]$ and $[Q G]$ are same to Eqs. (24) and (25), but $[M G],[N G]$ and $[O G]$ are different from Eqs. (21)-(23), which are as follows:

$$
\begin{aligned}
& {[M G]_{i j}}
\end{aligned}
$$

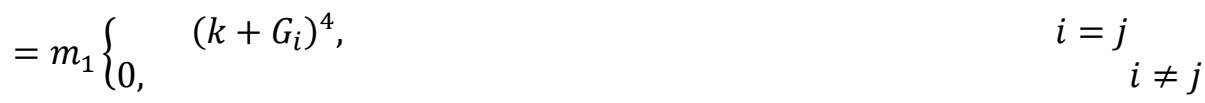

$[N G]_{i j}$

$=n_{1}\{0$,

$$
\left[1+\mu^{2}\left(k+G_{i}\right)^{2}\right]\left(k+G_{i}\right)^{2}, \quad i=j
$$

$[O G]_{i j}$

$=o_{1}\{0$,

$$
1+\mu^{2}\left(k+G_{i}\right)^{2}
$$

$i=j$

$i \neq j$

By substituting Eqs. (24), (25), (28)-(30) to (20), the band structure of such a nanobeam is finally calculated and obtained.

\section{LR elastic PC Euler nonlocal nanobeam}

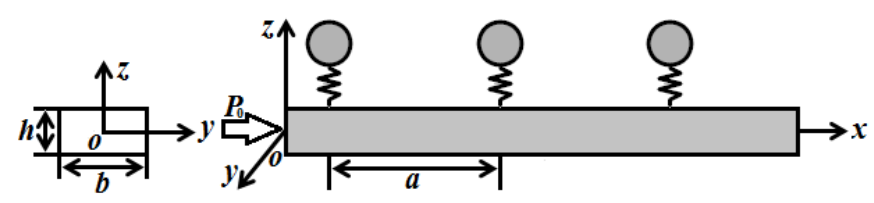

FIG. 3. Model of LR elastic PC nanobeam.

If the piezoelectric material PZT-4 is ignored and only elastic material epoxy is considered in the base beam, a LR elastic PC nanobeam is formed and shown in Fig. 3.

The corresponding bending vibration equations of such a nanobeam can be reduced from Eq. (1) and written as:

$$
\left\{\begin{array}{c}
m_{2} \frac{\partial^{4} W(x)}{\partial x^{4}}-n_{2} \frac{\partial^{2} W(x)}{\partial x^{2}}+\mu^{2} n_{2} \frac{\partial^{4} W(x)}{\partial x^{4}}-\omega^{2} o_{2} W(x)+\omega^{2} \mu^{2} o_{2} \frac{\partial^{2} W(x)}{\partial x^{2}}=\sum_{X} Q(X) \\
-\omega^{2} m_{\mathrm{R}} W_{\mathrm{R}}(X)=Q_{\mathrm{R}}(X)
\end{array}\right.
$$

By substituting Eqs. (2), (12), (13), (14), (17) and (18) to (31), it gives:

$$
\left\{\begin{array}{c}
\left\{m_{2}\left(k+G^{\prime}\right)^{4}+\left[1+\mu^{2}\left(k+G^{\prime}\right)^{2}\right] n_{2}\left(k+G^{\prime}\right)^{2}\right\} W\left(G^{\prime}\right)-\omega^{2}\left[1+\mu^{2}\left(k+G^{\prime}\right)^{2}\right] o_{2} W\left(G^{\prime}\right) \\
=-\frac{k_{\mathrm{R}}}{a}\left[\sum_{G^{\prime}} W\left(G^{\prime}\right)-W_{\mathrm{R}}(0)\right] \\
-\omega^{2} m_{\mathrm{R}} W_{\mathrm{R}}(0)=k_{\mathrm{R}}\left[\sum_{G^{\prime}} W\left(G^{\prime}\right)-W_{\mathrm{R}}(0)\right]
\end{array}\right.
$$

Eq. (32) can also be matriculated to the same form of Eq. (20), the relevant submatrices $[P G]$ and $[Q G]$ are same to Eqs. (24) and (25), but $[M G],[N G]$ and $[O G]$ are different from Eqs. (21)-(23), which are as follows: 


$$
\begin{aligned}
& {[M G]_{i j}}
\end{aligned}
$$

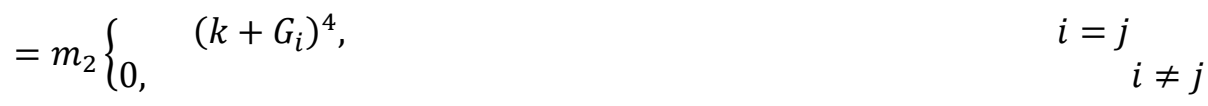

$$
\begin{aligned}
& {[N G]_{i j}} \\
& =n_{2}\left\{\begin{array}{l}
{\left[1+\mu^{2}\left(k+G_{i}\right)^{2}\right]\left(k+G_{i}\right)^{2}, \quad i=j \quad i \neq j}
\end{array}\right. \\
& {[O G]_{i j}} \\
& =o_{2}\left\{\begin{array}{l}
1+\mu^{2}\left(k+G_{i}\right)^{2}, \\
0,
\end{array} \quad i=j\right.
\end{aligned}
$$

By substituting Eqs. (24), (25), (33)-(35) to (20), the band structure of such a nanobeam is finally calculated and obtained.

\section{NUMERICAL RESULTS AND ANALYSES}

\section{A. Band structure corresponding to LR elastic PC Euler nonlocal nanobeam}

The band structure corresponding to a LR elastic PC Euler nonlocal nanobeam is shown in Fig. 4(a). During the calculation, nonlocal parameter $\eta=0.1$, number of reciprocal-lattice vectors $N=(2 \times 50+1)$, the parameters of each "spring-mass" resonator are: mass $m_{\mathrm{R}}=1 \times 10^{-18} \mathrm{~kg}$ and stiffness $k_{\mathrm{R}}=1 \times 10^{2} \mathrm{~N} / \mathrm{m}$. Besides, all of the geometric parameters are: width and height $b=h=10 \mathrm{~nm}$, lattice constant $a=100 \mathrm{~nm}$. Under $10 \mathrm{GHz}$, four complete band gaps with total width $5.7 \mathrm{GHz}$ and opening rate $57 \%$ can be opened (labeled by gray in Fig. 4(a)), which are respectively $0.1-1.7 \mathrm{GHz}$ with width $1.6 \mathrm{GHz}, 2.6-4.1 \mathrm{GHz}$ with width $1.5 \mathrm{GHz}, 5.6-7.2 \mathrm{GHz}$ with width $1.6 \mathrm{GHz}$ and $9-10 \mathrm{GHz}$ with width $1 \mathrm{GHz}$. As a comparison, Fig. 4(b) also gives the band structure of a pure elastic Euler nonlocal nanobeam with resonators ignored. Here, all the calculating parameters except for $m_{\mathrm{R}}=0 \mathrm{~kg}$ and $k_{\mathrm{R}}=0 \mathrm{~N} / \mathrm{m}$ are same to those in Fig. 4(a). As shown, if the homogeneous nanobeam is formed by elastic material epoxy without resonators attached, band gap cannot be opened in the corresponding band structure. Consequently, the application of resonators leads the original consecutive band structure of pure elastic Euler nanobeam to be effectively opened several complete wide band gaps with high opening rate.

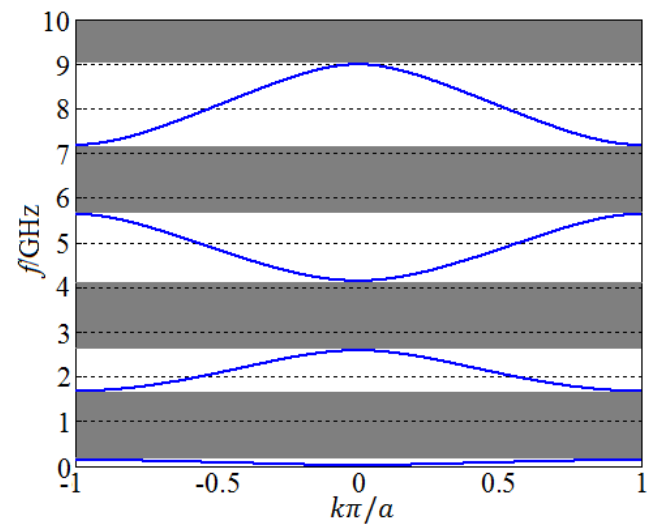

(a)

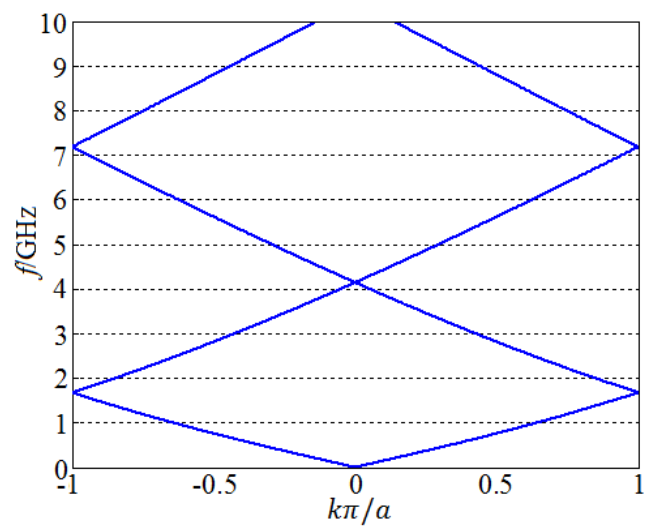

(b)

FIG. 4. Band structures corresponding to (a) LR elastic PC Euler nonlocal nanobeam and (b) pure elastic Euler nonlocal nanobeam, respectively.

\section{B. Band structure corresponding to LR piezoelectric PC Euler nonlocal nanobeam}




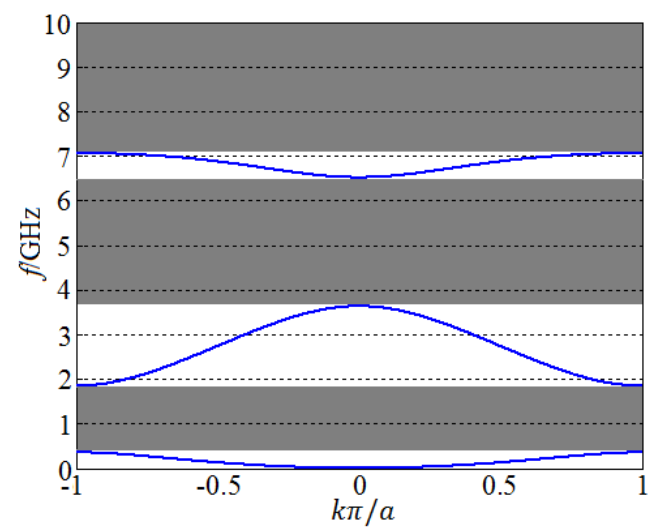

(a)

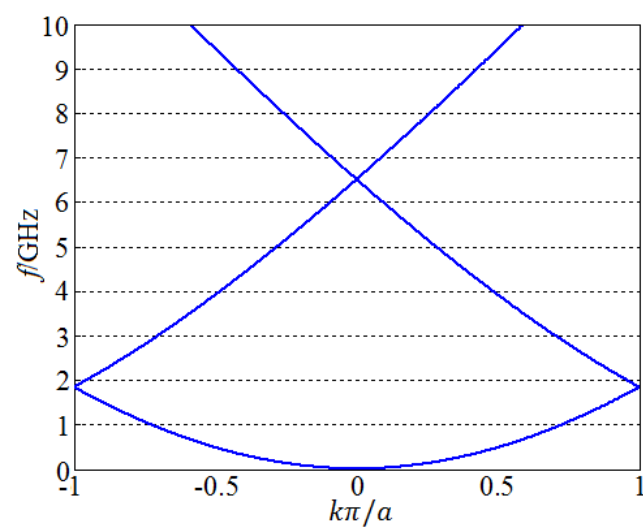

(b)

FIG. 5. Band structures corresponding to (a) LR piezoelectric PC Euler nonlocal nanobeam and (b) pure piezoelectric Euler nonlocal nanobeam, respectively.

The band structure corresponding to a LR piezoelectric PC Euler nonlocal nanobeam is shown in Fig. 5(a). During the calculation, the parameters of resonator and geometry are same to those in Fig. 4(a). Besides, the parameters of coupling fields between electricity and mechanics introduced by piezoelectric material are: $P_{0}=1 \times 10^{-8} \mathrm{~N}$ and $V=0.2 \mathrm{~V}$. As shown, three complete band gaps with total width $7.2 \mathrm{GHz}$ and opening rate $72 \%$ are opened less than $10 \mathrm{GHz}$, which are respectively $0.4-1.8 \mathrm{GHz}$ with width $1.4 \mathrm{GHz}, 3.6-6.5 \mathrm{GHz}$ with width $2.9 \mathrm{GHz}$ and 7.1-10GHz with width $2.9 \mathrm{GHz}$. As a comparison, Fig. 5(b) also gives the band structure of a pure piezoelectric Euler nonlocal nanobeam with resonators removed. Here, all the calculating parameters except for $m_{\mathrm{R}}=0 \mathrm{~kg}$ and $k_{\mathrm{R}}=0 \mathrm{~N} / \mathrm{m}$ are same to those in Fig. 5(a). Fig. 5(b) shows that if the homogeneous nanobeam is formed by piezoelectric material PZT-4 without resonators attached, band gap cannot be opened in the corresponding band structure. Hence, the original continuous band structure can be effectively divided into several complete wide band gaps under the resonators' help. In addition, the comparison of frequency range under $10 \mathrm{GHz}$ between Figs. 4(a) and 5(a) illustrates that wider band gaps with less quantity can be opened in piezoelectric nanobeam than elastic nanobeam. What's better, the studies on electro-mechanical coupling band gaps of such a piezoelectric nanobeam are applicable to the field of active control and intelligentialize in NEMS.

\section{Band structures corresponding to LR elastic/piezoelectric PC Euler nonlocal nanobeams}

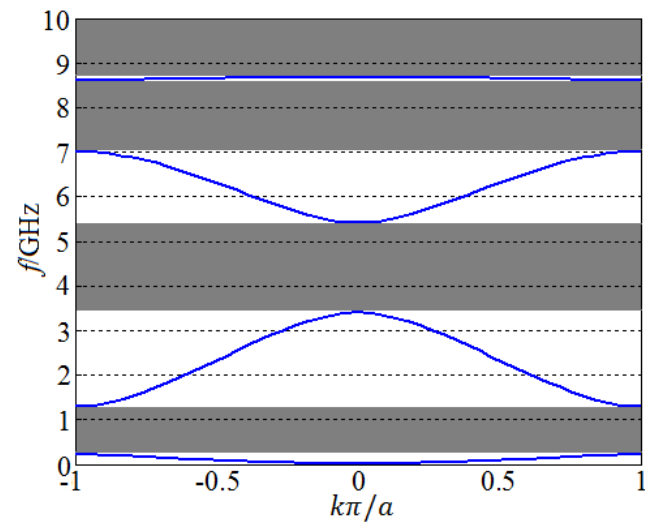

(a)

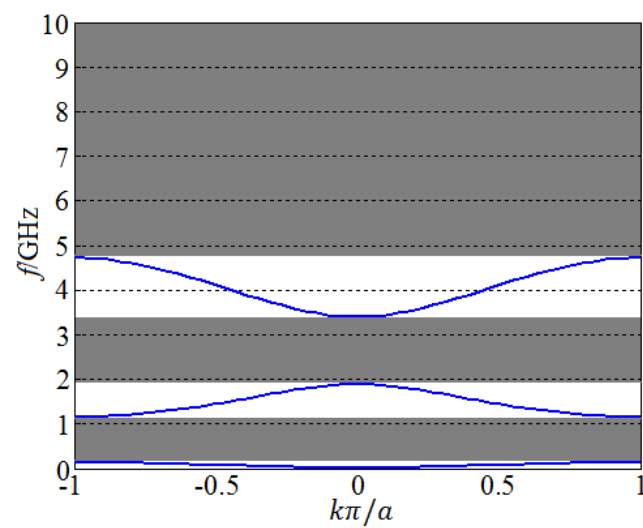

(b) 


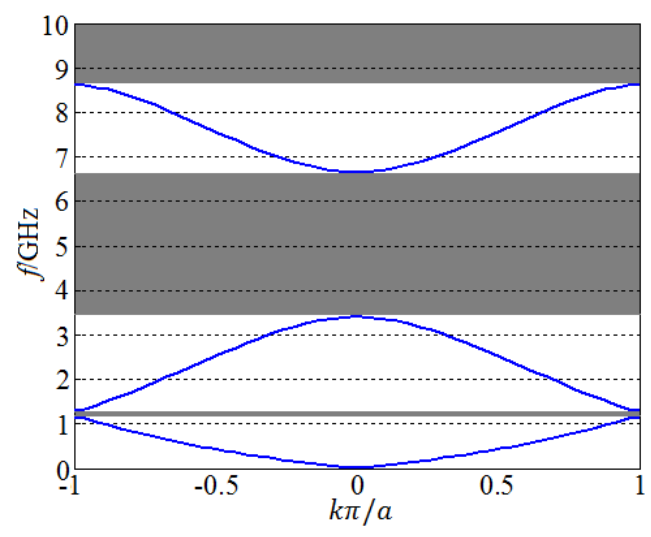

(c)

FIG. 6. Band structures corresponding to LR elastic/piezoelectric PC Euler nonlocal nanobeams with "spring-mass" resonators periodically attached on (a) piezoelectric material PZT-4, (b) elastic material epoxy and (c) with resonators removed, separately.

Fig. 6(a) and 6(b) show respectively the band structures corresponding to LR elastic/piezoelectric PC Euler nonlocal nanobeams with "spring-mass" resonators periodically attached on piezoelectric material PZT-4 and elastic material epoxy. In addition, Fig. 6(c) also gives the band structure corresponding to an elastic/piezoelectric PC Euler nonlocal nanobeam without attaching resonators. Here, the geometric parameters used in the calculations are: $a_{1}=$ $a_{2}=50 \mathrm{~nm}, a=a_{1}+a_{2}=100 \mathrm{~nm}$ and $b=h=10 \mathrm{~nm}$. Besides, the parameters of resonator and electro-mechanical coupling fields are same to those in Fig. 5. As shown in Fig. 6(a), four complete band gaps with total width $6 \mathrm{GHz}$ and opening rate $60 \%$ can be opened under $10 \mathrm{GHz}$, which are respectively $0.2-1.3 \mathrm{GHz}$ with width $1.1 \mathrm{GHz}, 3.4-5.4 \mathrm{GHz}$ with width $2 \mathrm{GHz}, 7-8.6 \mathrm{GHz}$ with width $1.6 \mathrm{GHz}$ and $8.7-10 \mathrm{GHz}$ with width $1.3 \mathrm{GHz}$. As shown in Fig. 6(b), three complete band gaps with total width $7.9 \mathrm{GHz}$ and opening rate $79 \%$ can be opened under $10 \mathrm{GHz}$, which are respectively $0.1-1.2 \mathrm{GHz}$ with width $1.1 \mathrm{GHz}, 1.9-3.4 \mathrm{GHz}$ with width $1.5 \mathrm{GHz}$ and $4.7-10 \mathrm{GHz}$ with width $5.3 \mathrm{GHz}$. As shown in Fig. 6(c), three complete band gaps with total width $4.8 \mathrm{GHz}$ and opening rate $48 \%$ can be opened under $10 \mathrm{GHz}$, which are respectively $1.2-1.3 \mathrm{GHz}$ with width $0.1 \mathrm{GHz}, 3.4-6.7 \mathrm{GHz}$ with width $3.3 \mathrm{GHz}$ and $8.6-10 \mathrm{GHz}$ with width $1.4 \mathrm{GHz}$. By comparing Figs. $6(\mathrm{a})$ and $6(\mathrm{~b})$, if resonators are attached on epoxy, band gaps less than $10 \mathrm{GHz}$ with higher opening rate can be opened. By comparing Figs. 6(a) (or 6(b)) and 6(c), band gaps less than 10GHz with higher opening rate can be opened in the elastic/piezoelectric nanobeam with resonators attached than that with resonators removed. By comparing 4(a), 5(a) and 6(b), band gaps less than $10 \mathrm{GHz}$ with higher opening rate in the nanobeam with base beam composed of two materials can be opened than those with base beam composed of only one material. Moreover, by comparing Figs. 4(b), 5(b) and 6(c), several band gaps with almost half frequency range under 10GHz are opened in the nanobeam composed by two different materials, but no band gap is opened in the pure non-LR Euler nonlocal nanobeams by only one material.

As shown in Figs. 4, 5 and 6, widest band gaps can be opened in the elastic/piezoelectric nanobeam with epoxy attached by resonators, which is more appropriate for the application in engineering. Thus, the starting frequencies and widths of band gaps of first order (1st BG $f_{\mathrm{s}}$ and 1st BG $f_{\mathrm{w}}$ ), second order (2nd BG $f_{\mathrm{s}}$ and 2nd BG $f_{\mathrm{w}}$ ) and third order (3rd BG $f_{\mathrm{s}}$ and 3rd BG $f_{\mathrm{w}}$ ), as well as total bandgap width of first three orders (T_BG $f_{\mathrm{w}}$ ) of such a nanobeam are applied to study the influencing rules of coupling fields between electricity and mechanics, 
"spring-mass" resonators, nonlocal effect and different geometric parameters on band gaps in detail.

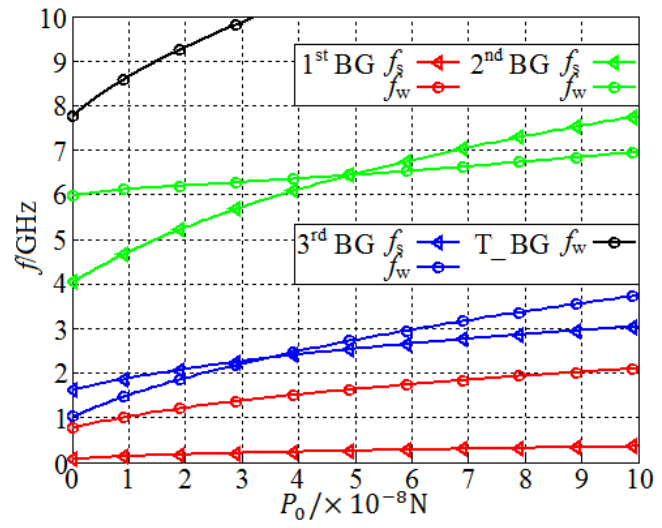

(a)

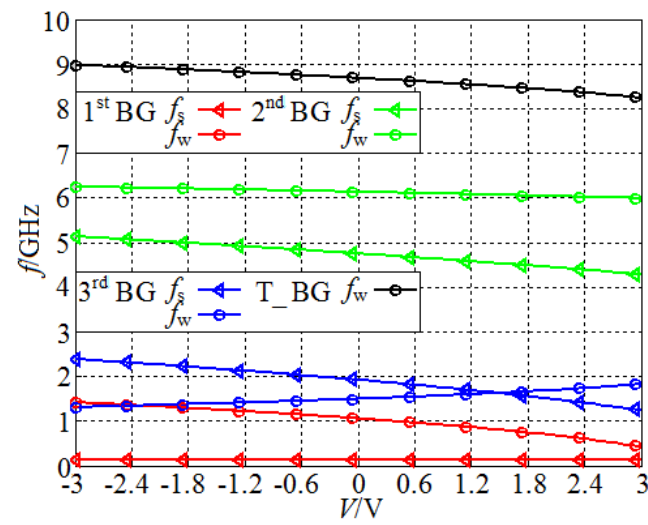

(b)

FIG. 7. Influences of (a) $P_{0}$ and (b) $V$ on starting frequencies, widths and total width of band gaps of first three orders, respectively.

Figs. 7(a) and 7(b) display the influences of $P_{0}$ and $V$ on starting frequencies, widths and total width of band gaps of first three orders, respectively. During the calculations, all the other parameters except for $P_{0}$ and $V$ are same to those in Fig. 6(b), the region of $P_{0}$ is $0 \times 10^{-8}-$ $10 \times 10^{-8} \mathrm{~N}$, and the range of $V$ is $-3-3 \mathrm{~V}$. As displayed in Fig. 7(a), with the increase of $P_{0}$, all of the starting frequencies of band gaps of first three orders keep increasing. Besides, the growth rate increases with the increase of bandgap order. By increasing $P_{0}$, all the three bandgap widths also keep on increasing, which finally makes the total bandgap width of first three orders also keep on increasing. Differently, with the increase of $V$, all of the starting frequencies of band gaps of first three orders keep on decreasing, the bandgap widths of first and third orders keep on decreasing but that of second order keeps on increasing, which finally leads the total bandgap width of first three orders to keep on decreasing.

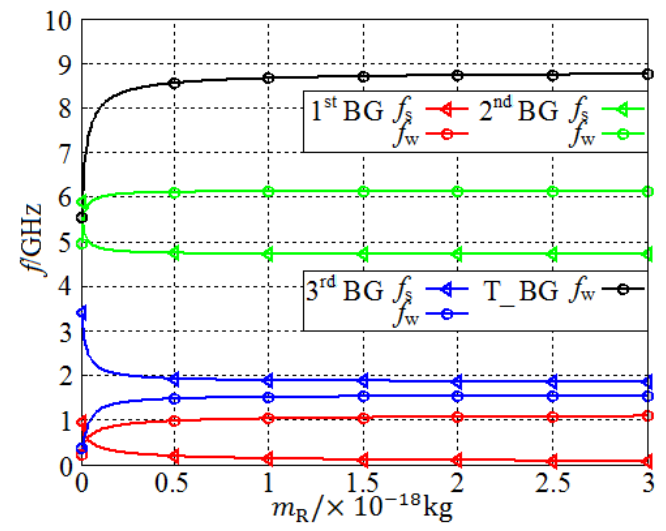

(a)

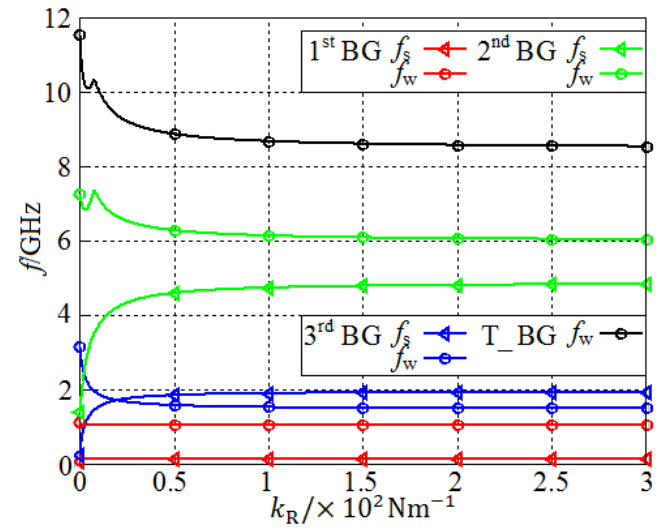

(b)

FIG. 8. Influences of (a) $m_{\mathrm{R}}$ and (b) $k_{\mathrm{R}}$ on starting frequencies, widths and total width of band gaps of first three orders, respectively.

Figs. 8(a) and 8(b) give the influences of $m_{\mathrm{R}}$ and $k_{\mathrm{R}}$ on starting frequencies, widths and total width of band gaps of first three orders, respectively. During the calculations, the region of $m_{\mathrm{R}}$ is $0 \times 10^{-18}-3 \times 10^{-18} \mathrm{~kg}$, the region of $k_{\mathrm{R}}$ is $0 \times 10^{2}-3 \times 10^{2} \mathrm{~N} / \mathrm{m}$, and all the other parameters are same to those in Fig. 6(b). As shown in Figs. 8(a) and 8(b), all of the starting frequencies of band gaps of first three orders keep on increasing with the increase of $k_{\mathrm{R}}$ or the 
decrease of $m_{\mathrm{R}}$, which belongs to the typical influencing rules of LRPCs. Moreover, because the "spring-mass" resonators only affect limited frequency region of each band depending on the parameters of resonator and base beam, all the starting frequencies are almost unaffected if $m_{R}$ and $k_{\mathrm{R}}$ are much larger as shown. Besides, as shown in Fig. 8(a), with the increase of $m_{\mathrm{R}}$, all the widths and total width of band gaps of first three orders keep on sharply increasing in the first place and then nearly constant. As shown in Fig. 8(b), $k_{\mathrm{R}}$ almost has nothing to do with the first bandgap width. With the increase of $k_{\mathrm{R}}$, the second bandgap width keeps decreasing sharply at first and then nearly constant, the third bandgap width also keeps decreasing sharply at first and then nearly constant after a up and down, which leads the total width of band gaps of first three orders appear the same variation trend.

Fig. 9 display the influences of $\eta$ on starting frequencies, widths and total width of band gaps of first three orders. Here, all the calculating parameters except for $\eta$ are identical to those in Fig. 6(b), and the range of $\eta$ is from 0 to 0.5 . As shown, by increasing $\eta$, the starting frequencies of band gaps of first and third orders keep on decreasing, but that of the second order keeps on increasing at first and then decreasing. Besides, with the increase of $\eta$, all the widths of band gaps of first three orders keep decreasing, which finally leads the total width of band gaps of first three orders keep on decreasing. Moreover, with the increase of bandgap order, the starting frequency and width of band gap are affected more obviously. Hence, the nonlocal parameter introduced by the nanoscale can shorten the bandgap widths.

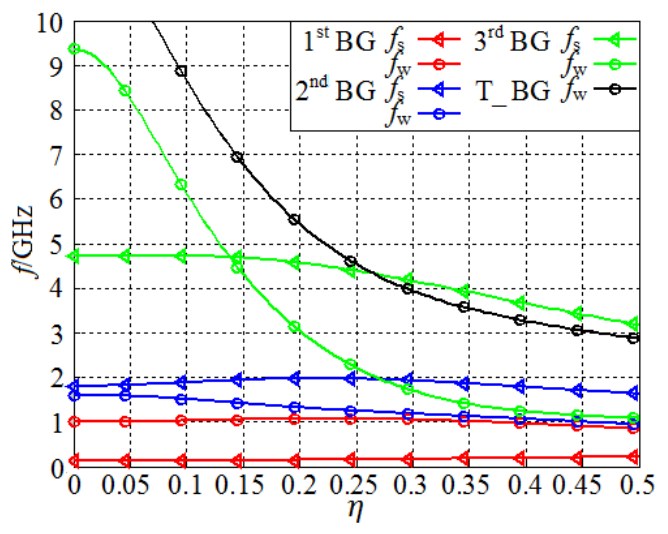

FIG. 9. Influences of $\eta$ on starting frequencies, widths and total width of band gaps of first three orders.

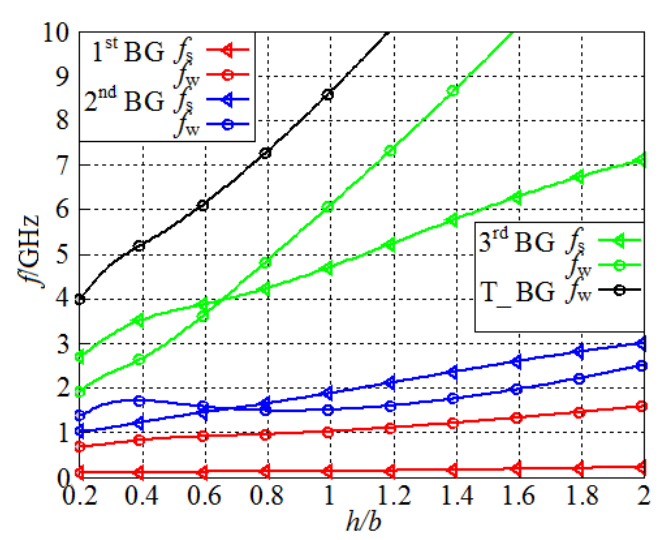

(a)

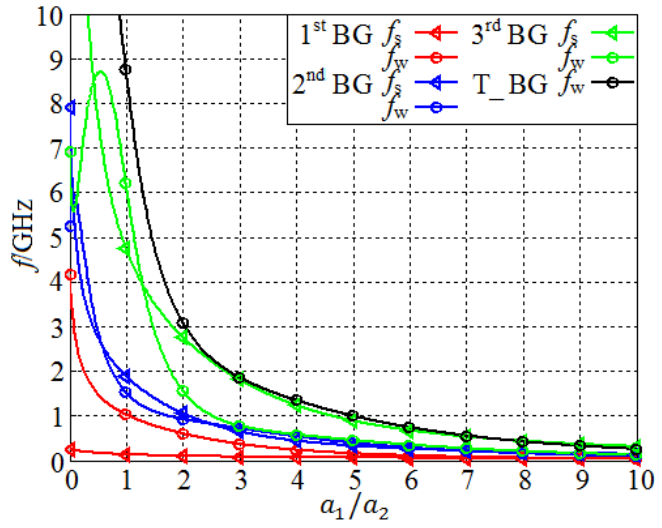

(b)

FIG. 10. Influences of (a) $h / b$ and (b) $a_{1} / a_{2}$ on starting frequencies, widths and total width of band gaps of first three orders, respectively. 
Figs. 10(a) and 10(b) show the influences of ratios $h / b$ and $a_{1} / a_{2}$ on starting frequencies, widths and total width of band gaps of first three orders, respectively. During the calculations, the region of $h / b$ is $0.2-2$ with the constant $b=10 \mathrm{~nm}$, the range of $a_{1} / a_{2}$ is from 0 to 10 with the constant $a_{2}=50 \mathrm{~nm}$, and all the other parameters are identical to those in Fig. 6(b). Fig. 10(a) shows that all the starting frequencies of band gaps of first three orders keep on increasing with the increase of $h / b$. Besides, the growth rate increases with the increase of bandgap order. Besides, with the increase of $h / b$, the widths of band gaps of first and third orders keep on increasing, and that of the second order keeps on increasing after a up and down, which finally leads the total width of band gaps of first three orders to keep on increasing. Consequently, band gaps with wider width are opened with larger height than width. As shown in Fig. 10(b), by increasing $a_{1} / a_{2}$, all the starting frequencies of band gaps of first three orders keep on going down to be almost zero. However, by increasing $a_{1} / a_{2}$, the width of band gap of first order keeps on going down to be almost zero, that of the second order increases firstly and then keeps on going down to be almost zero, and that of the third order decreases at first, then increases and lastly keeps going down to be close to zero. If $a_{1}$ is much larger than $a_{2}$, the nanobeam is considered to be composed by only one material, that is why the band gaps are not easy to be opened with the high ratio $a_{1} / a_{2}$. Consequently, in order to meet requirement of band gaps as wide as possible, the reasonable ratios $a_{1} / a_{2}$ and $h / b$ should be picked.

\section{CONCLUSIONS}

In this paper, the band structures of three different LRPC Euler nonlocal nanobeams are calculated and compared with the application of PWE method. Particularly, the bandgap properties of LR elastic/piezoelectric PC Euler nonlocal nanobeam with epoxy periodically attaching "spring-mass" resonators, which the opening rate is the highest, are revealed minutely. The main conclusions are summarized as follows:

1. By increasing the external axial force or decreasing the external electrical voltage introduced by the electro-mechanical coupling fields, the total width of band gaps of first three orders keep on increasing. Besides, the starting frequencies and widths of band gaps of first three orders can be effectively adjusted by the electro-mechanical coupling fields, which plays an active role in the further application in the field of active control on ultrahigh vibration.

2. By increasing the mass or decreasing the stiffness introduced by the "spring-mass" resonator, the widths and total width of band gaps of first three orders keep increasing, but the adjustable region is limited. Oppositely, with the increase of stiffness or the decrease of mass, the starting frequencies increase, this is accord with the influencing rules of resonators.

3. Generally, by increasing the nonlocal parameter introduced by nanoscale, all the starting frequencies, widths and total width of band gaps of first three orders keep on decreasing.

4. Band gaps with wider width are opened with larger height than width and longer length of piezoelectric material than that of elastic material. Band gaps are harder to be opened with longer length of elastic material than piezoelectric material.During the process of design, the reasonable geometric parameters should be selected to meet the requirement of starting frequencies and widths of band gaps.

\section{Availability of data and materials}

Not applicable.

\section{Competing interests}


The authors declare that they have no competing interests.

\section{Funding}

Supported by National Natural Science Foundation of China (Grant No. 11847009).

\section{Authors' contributions}

DQ proposed the model and numerical method, JW and FH analyzed the data, all the authors wrote and improved the paper. All authors read and approved the final manuscript.

\section{Acknowledgements}

Not applicable.

\section{REFERENCES}

[1] Verd J, Segura J. Editorial for the Special Issue on Development of CMOS-MEMS/NEMS Devices[J]. Micromachines, 2019, 10(4): 273.

[2] Li S B, Dou Y H, Chen T N, et al. Designing a broad locally-resonant bandgap in a phononic crystals[J]. Physics Letters A, 2019, 383(12): 1371-1377.

[3] Chen A L, Yan D J, Wang Y S, et al. Anti-plane transverse waves propagation in nanoscale periodic layered piezoelectric structures[J]. Ultrasonics, 2016, 65: 154-164.

[4] Yan D J, Chen A L, Wang Y S, et al. Propagation of guided elastic waves in nanoscale layered periodic piezoelectric composites[J]. European Journal of Mechanics A/Solids, 2017, 66: 158-167.

[5] Yan D J, Chen A L, Wang Y S, et al. In-plane elastic wave propagation in nanoscale periodic layered piezoelectric structures[J]. International Journal of Mechanical Sciences, 2018, 142-143: 276-288.

[6] Miranda Jr E J P, Dos Santos J M C. Complete band gaps in nano-piezoelectric phononic crystals[J]. Materials Research, 2017, 20: 15-38.

[7] Qian D H. Bandgap properties of a piezoelectric phononic crystal nanobeam with surface effect[J]. Journal of Applied Physics, 2018, 124(5): 055101.

[8] Qian D H. Bandgap properties of a piezoelectric phononic crystal nanobeam based on nonlocal theory[J]. Journal of Materials Science, 2019, 54(5): 4038-4048.

[9] Qian D H, Shi Z Y, Ning C W, et al. Nonlinear bandgap properties in a nonlocal piezoelectric phononic crystal nanobeam[J]. Physics Letters A, 2019, 383(25): 3101-3107.

[10] Zeng S, Wang B L, Wang K F. Static stability analysis of nanoscale piezoelectric shells with flexoelectric effect based on couple stress theory[J]. Microsystem Technologies, 2018: 1-11.

[11] Zhou Y, Xu Y, Pan D, et al. Improved incorporation of strain gradient elasticity in the flexoelectricity based energy harvesting from nanobeams[J]. Physica E: Low-dimensional Systems and Nanostructures, 2018, 98: 148-158.

[12] Ansari R, Oskouie M F, Gholami R, et al. Thermo-electro-mechanical vibration of post buckled piezoelectric Timoshenko nanobeams based on the nonlocal elasticity theory[J]. Composites Part B, 2016, 89: 316-327.

[13] Arefi M, Rabczuk T. A nonlocal higher order shear deformation theory for electro-elastic analysis of a piezoelectric doubly curved nano shell[J]. Composites Part B: Engineering, 2019, 168: 496-510.

[14] Chuang K C, Yuan Z W, Guo Y Q, et al. A self-demodulated fiber Bragg grating for investigating impact-induced transient responses of phononic crystal beams[J]. Journal of Sound \& Vibration, 2018, 431: 40-53. 
[15] Qi X Q, Li T J, Zhang J L. Tuning characteristics of bandgap in a stub phononic crystal plate with slit and slider structure[J]. Materials Research Express, 2019, 6(12): 126206.

[16] Guo X, Wei P, Lan M, et al. Dispersion relations of elastic waves in one-dimensional piezoelectric/piezomagnetic phononic crystal with functionally graded interlayers.[J]. Ultrasonics, 2016, 70: 158-171.

[17] Sun W B, Wang T, Sun X W, et al. Defect states and vibration energy recovery of novel two-dimensional piezoelectric phononic crystal plate[J]. Acta Physica Sinica, 2019, 68(23): 234206.

[18] Deng T, Zhang S Z, Gao Y W. A magnetic-dependent vibration energy harvester based on the tunable point defect in 2D magneto-elastic phononic crystals[J]. Crystal, 2019, 9(5): 261.

[19] Yan Z, Wei C, Zhang C. Band structures of elastic SH waves in nanoscale multi-layered functionally graded phononic crystals with/without nonlocal interface imperfections by using a local RBF collocation method[J]. Acta Mechanica Solida Sinica, 2017, 30(4): 390-403.

[20] Hosseini S M, Zhang C Z. Nonlocal coupled thermoelastic wave propagation band structures of nano-scale phononic crystal beams based on GN theory with energy dissipation: An analytical solution[J]. Wave Motion, 2020, 92: 102429.

[21] Xiao Y, Wen J, Wen X. Flexural wave band gaps in locally resonant thin plates with periodically attached spring-mass resonators[J]. Journal of Physics D Applied Physics, 2012, 45(19): 195401-195412(12).

[22] Qian D H. Electro-mechanical coupling wave propagating in a locally resonant piezoelectric/elastic phononic crystal nanobeam with surface effects[J]. Applied Mathematics and Mechanics (English Edition), 2020, 41(3): 425-438. 
Figures

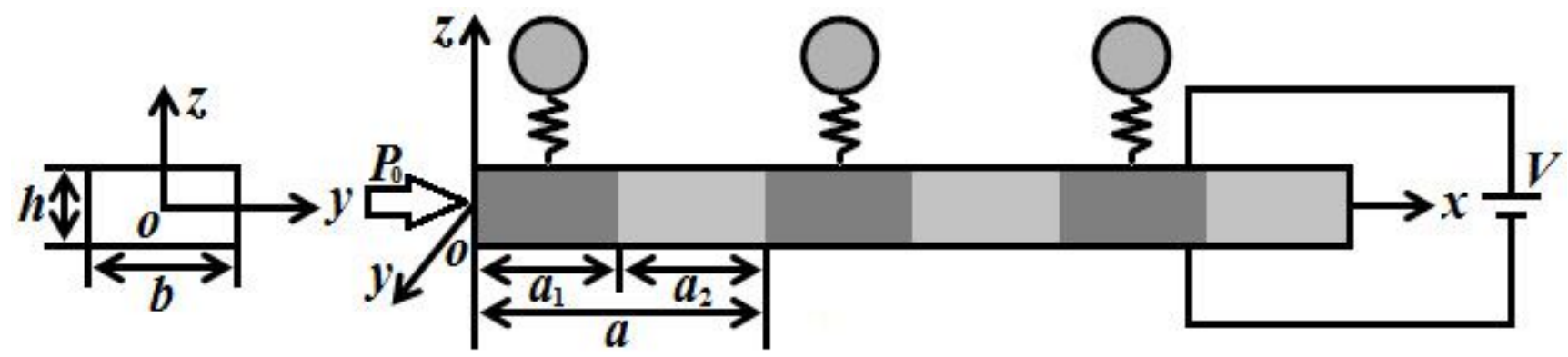

Figure 1

Model of LR elastic/piezoelectric PC nanobeam

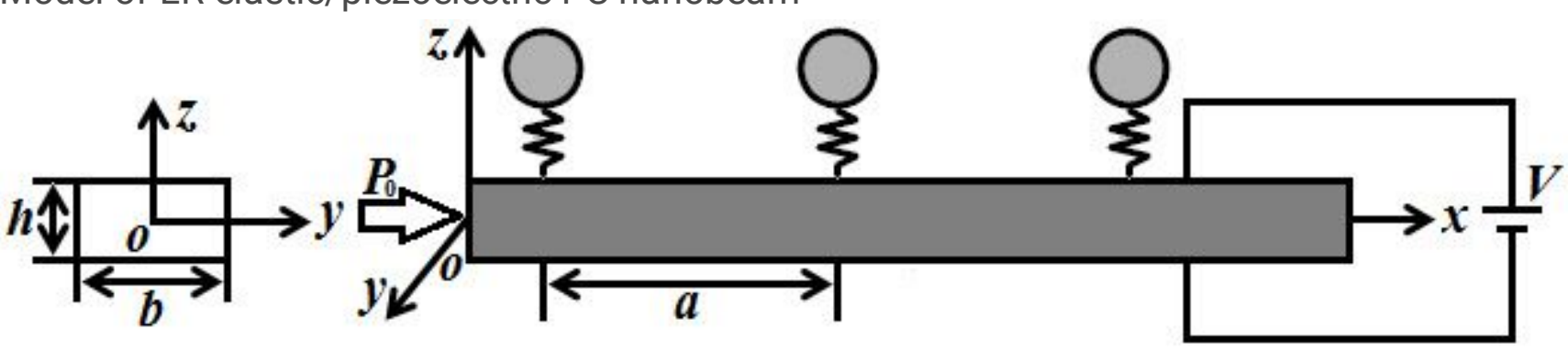

Figure 2

Model of LR piezoelectric $\mathrm{PC}$ nanobeam

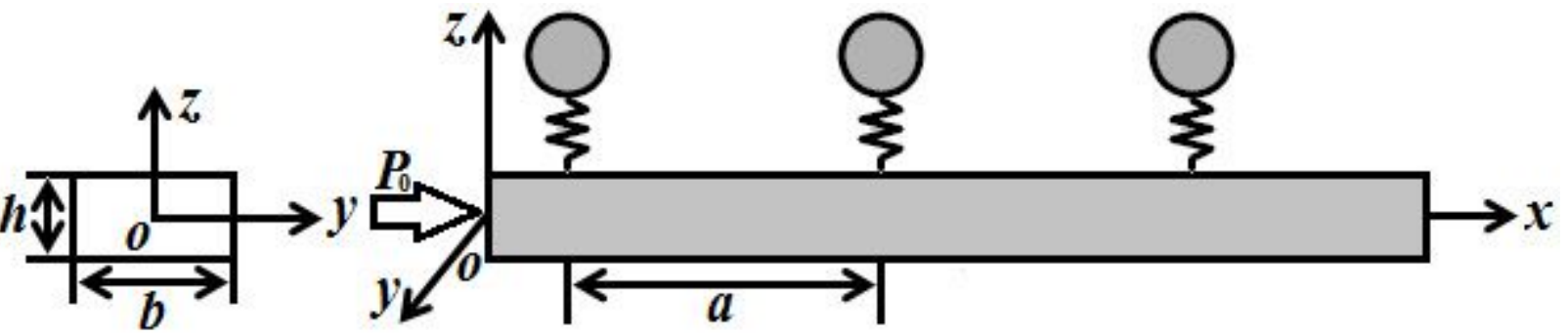

Figure 3

Model of LR elastic PC nanobeam. 


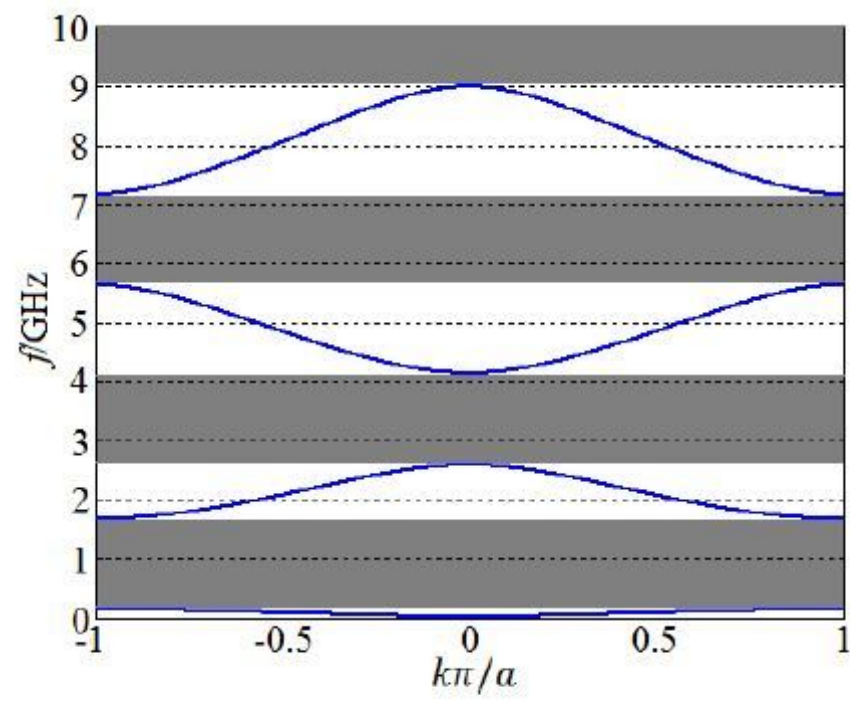

(a)

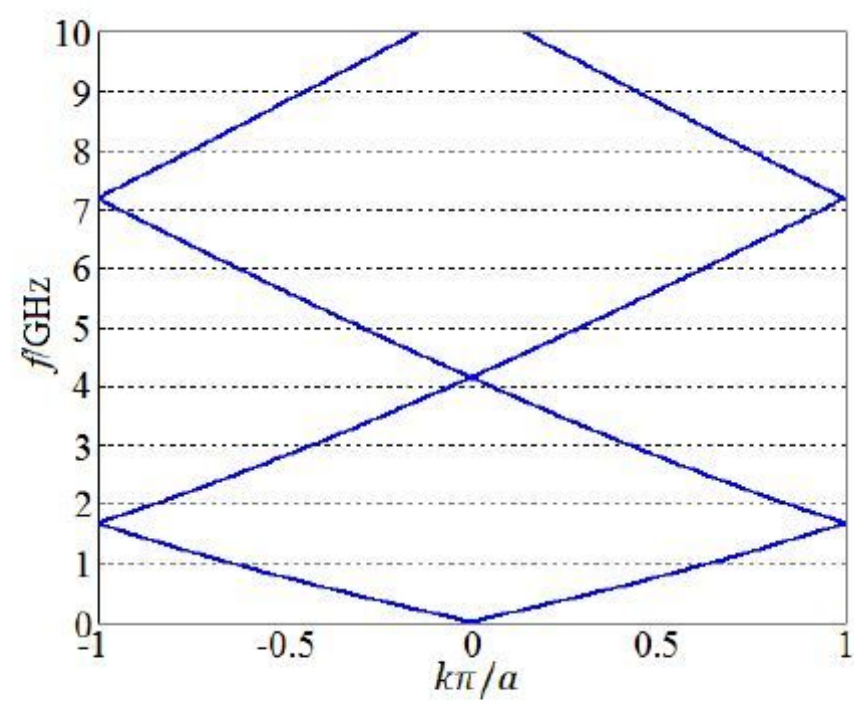

(b)

\section{Figure 4}

Band structures corresponding to (a) LR elastic PC Euler nonlocal nanobeam and (b) pure elastic Euler nonlocal nanobeam, respectively.

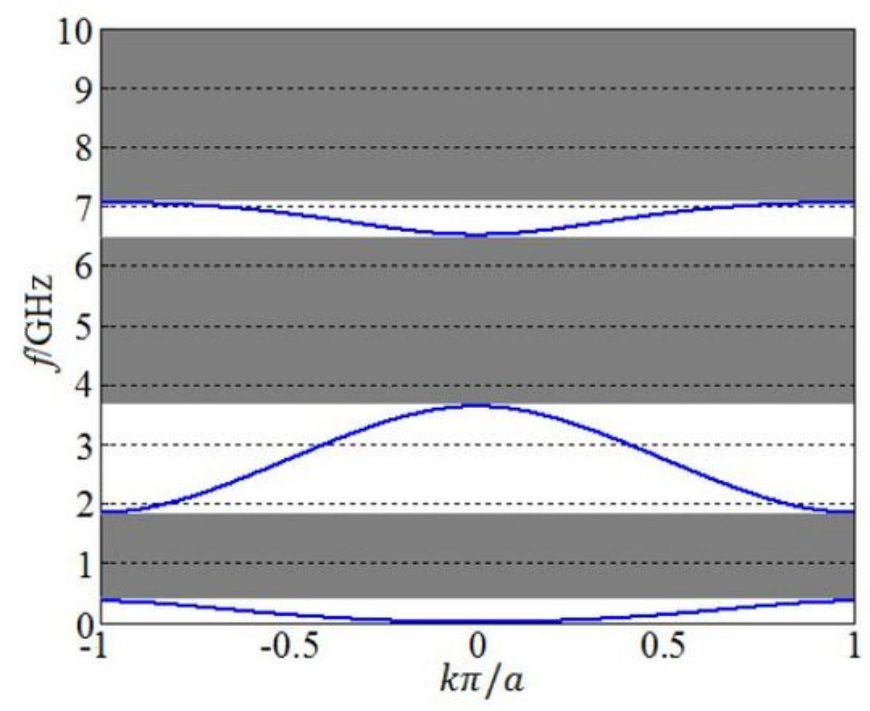

(a)

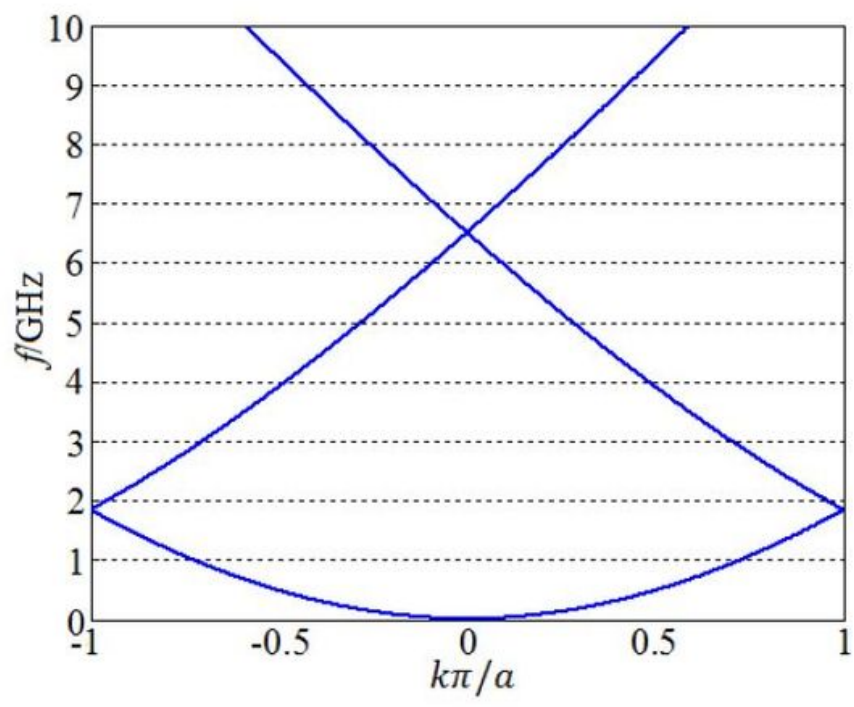

(b)

\section{Figure 5}

Band structures corresponding to (a) LR piezoelectric PC Euler nonlocal nanobeam and (b) pure piezoelectric Euler nonlocal nanobeam, respectively. 


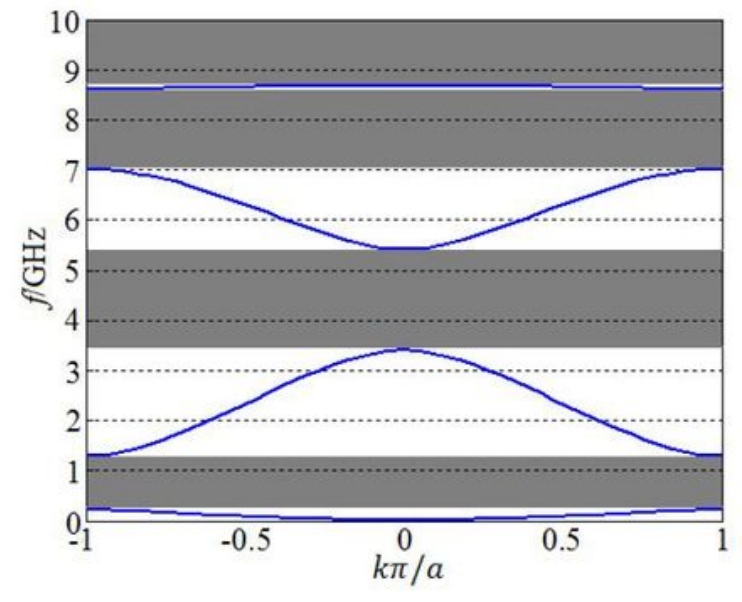

(a)

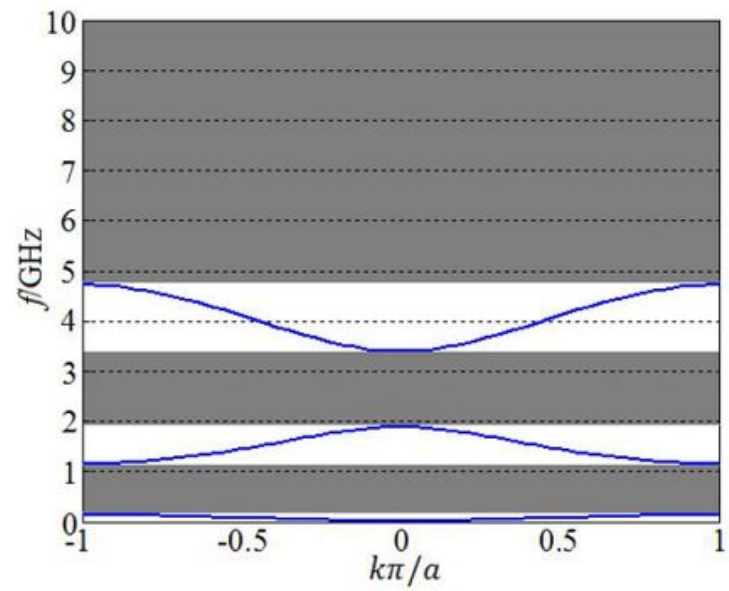

(b)

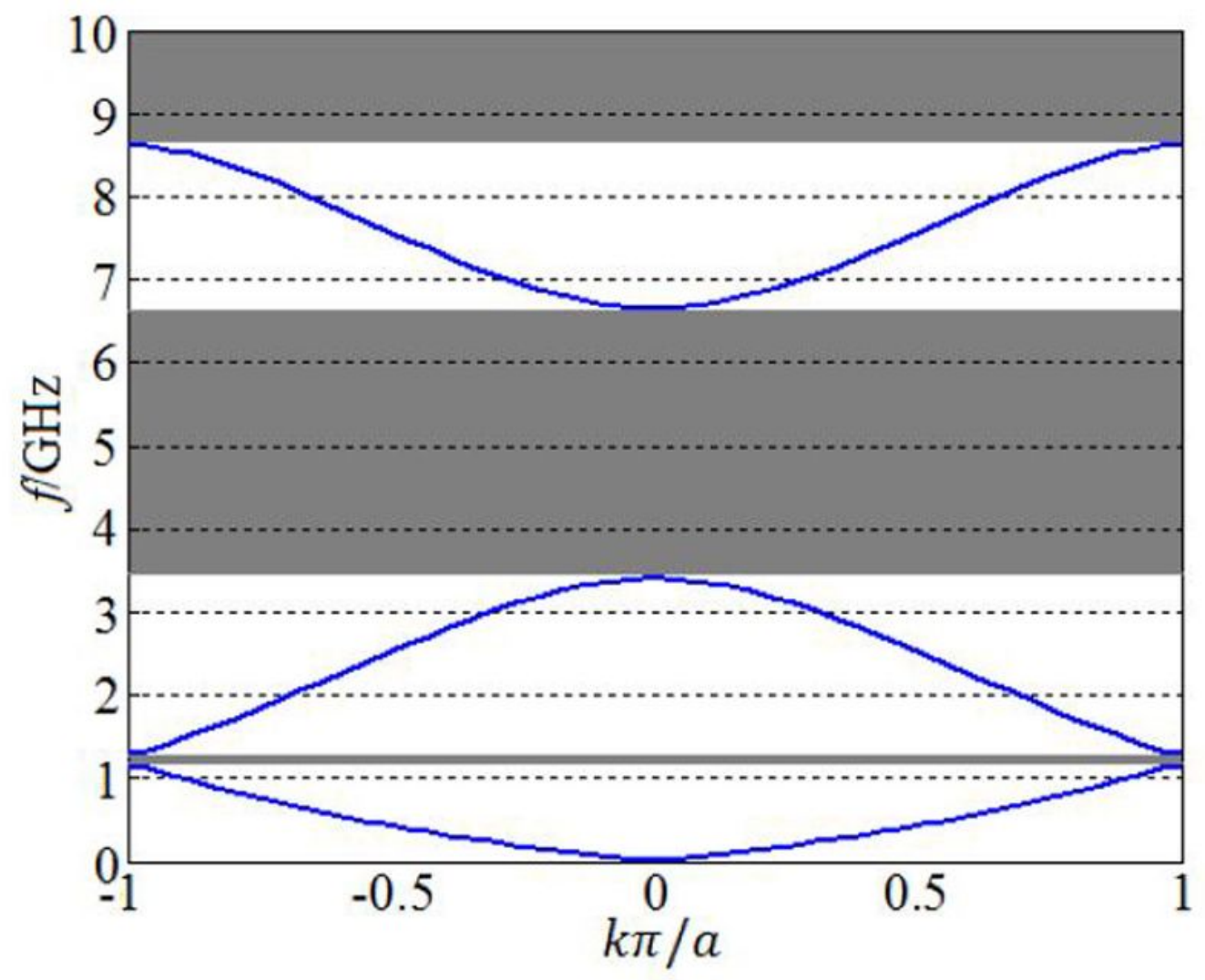

(c)

\section{Figure 6}

Band structures corresponding to LR elastic/piezoelectric PC Euler nonlocal nanobeams with "springmass" resonators periodically attached on (a) piezoelectric material PZT-4, (b) elastic material epoxy and (c) with resonators removed, separately. 


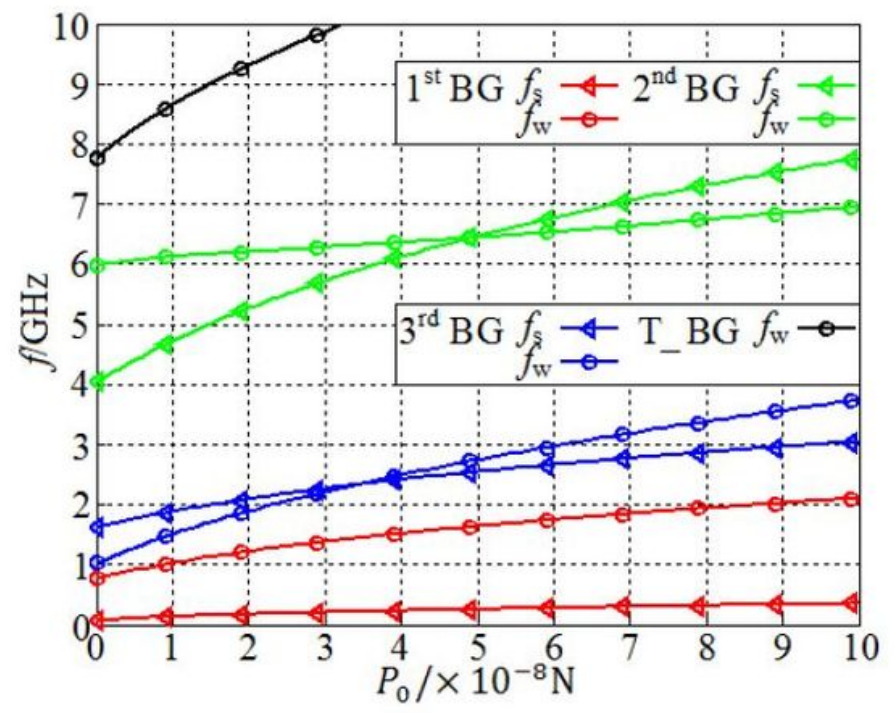

(a)

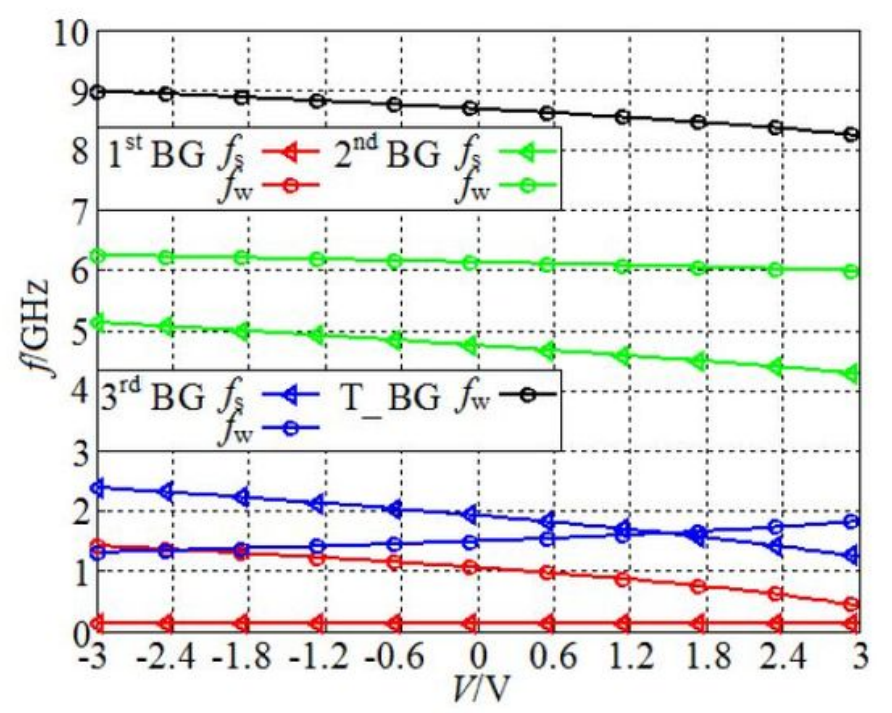

(b)

\section{Figure 7}

Influences of (a) $\nabla 0$ and (b) $\nabla$ on starting frequencies, widths and total width of band gaps of first three orders, respectively.

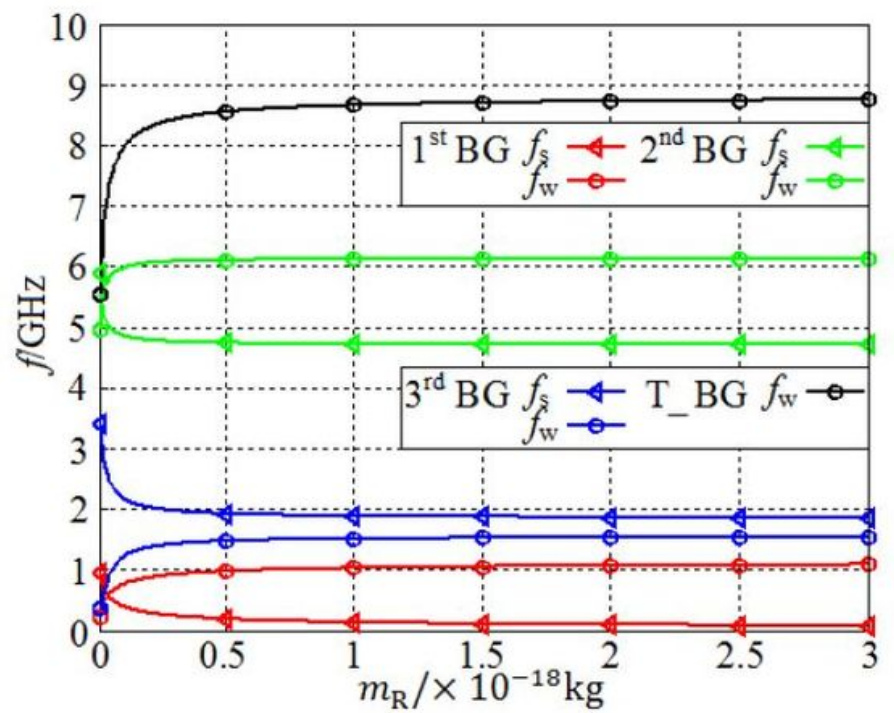

(a)

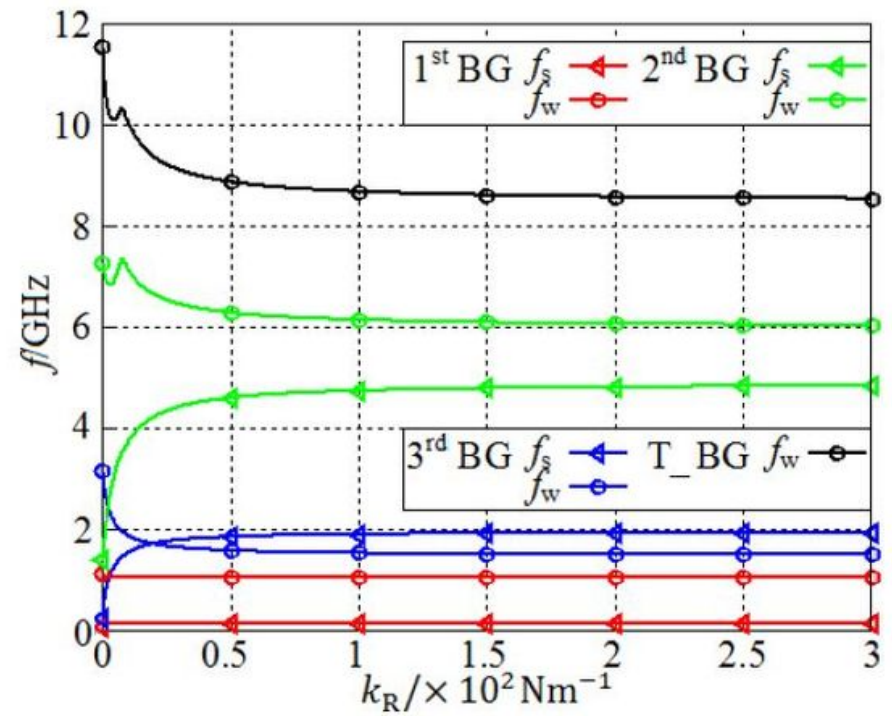

(b)

Figure 8

Influences of (a) $\triangle R$ and (b) $\otimes R$ on starting frequencies, widths and total width of band gaps of first three orders, respectively. 


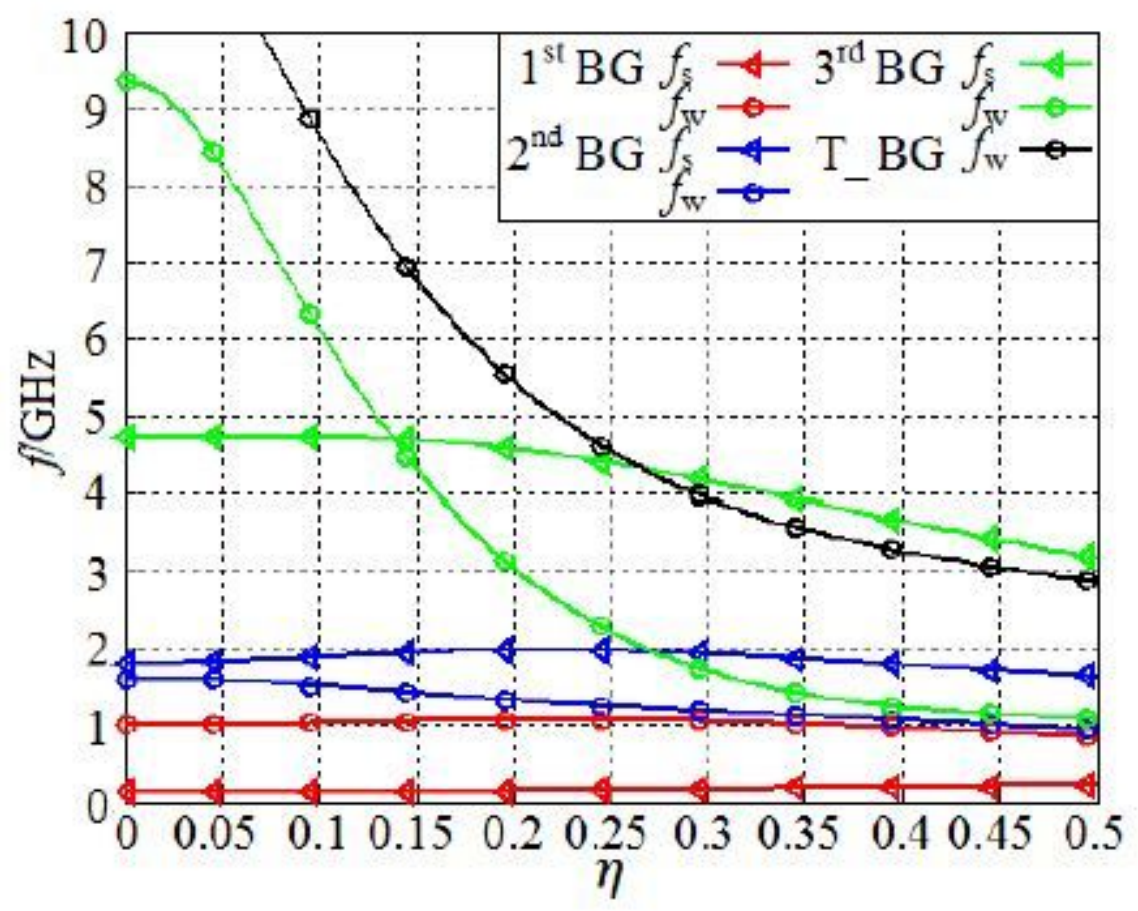

Figure 9

Influences of $\nabla$ on starting frequencies, widths and total width of band gaps of first three orders.

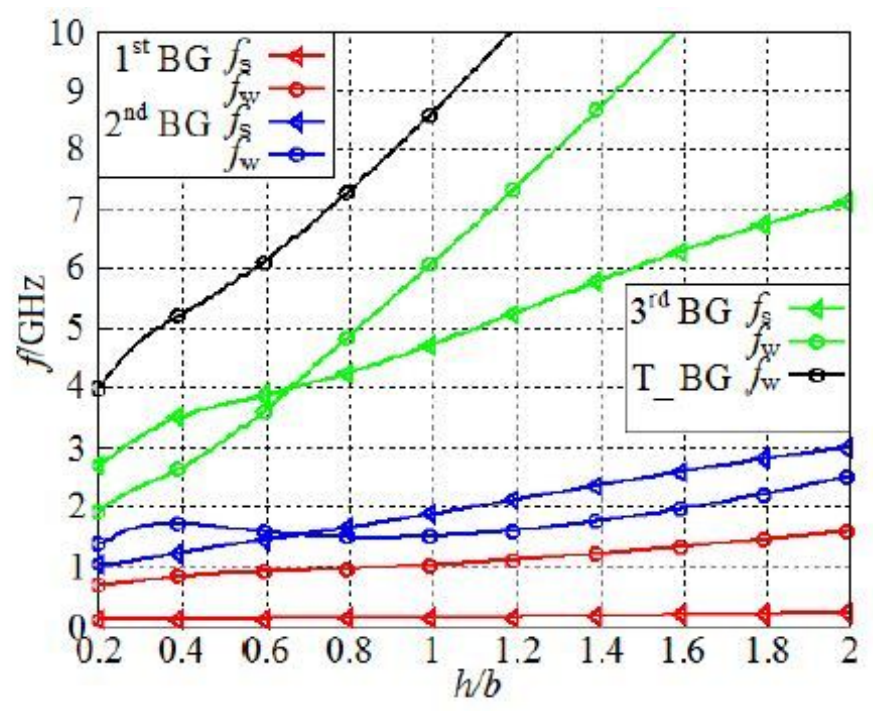

(a)

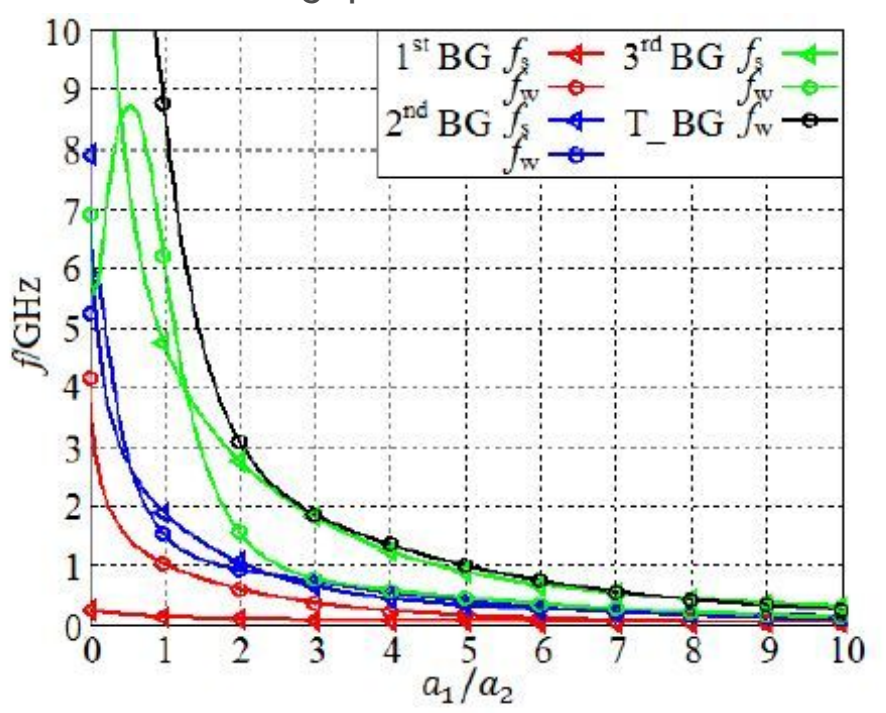

(b)

\section{Figure 10}

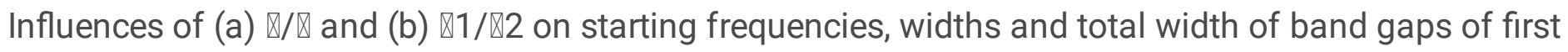
three orders, respectively. 